University of New Mexico

UNM Digital Repository

\title{
4-1-1979
}

\section{The genetical evolution of patterns of sexuality: Darwinian fitness.}

Eric Charnov

Follow this and additional works at: https://digitalrepository.unm.edu/biol_fsp

Part of the Biology Commons

\section{Recommended Citation}

Charnov, E.L. 1979. The genetical evolution of patterns of sexuality: Darwinian fitness. American Naturalist 113:465-480

This Article is brought to you for free and open access by the Academic Department Resources at UNM Digital Repository. It has been accepted for inclusion in Biology Faculty \& Staff Publications by an authorized administrator of UNM Digital Repository. For more information, please contact disc@unm.edu. 


\title{
THE \\ AMERICAN NATURALIST
}

Vol. 113, No. 4

The American Naturalist

April 1979

\section{THE GENETICAL EVOLUTION OF PATTERNS OF SEXUALITY: DARWINIAN FITNESS*}

\author{
ERIC L. Charnov
}

Department of Biology, University of Utah, Salt Lake City, Utah 84112

Several authors have recently provided valuable discussions on the evolution and implications of sexual reproduction (Trivers 1972; Williams 1975; Ghiselin 1974). The models proposed here concern one particular aspect of this broad topic, which may best be termed sex allocation. Most animal or plant species produce only two types of gametes (large, small). In hermaphroditic organisms, a single individual produces both large and small gametes in its lifetime. In dioecious organisms, males and females are separate throughout their lives. Hermaphroditism (in animals) takes two forms: (1) Sequential-an individual functions early in life as one sex and then switches to the other sex for the rest of its life ( $+\rightarrow$ o protogyny; $\hat{\sigma} \rightarrow+$ protandry); (2) Simultaneous - an individual produces both kinds of gametes in each breeding season. While this scheme is too simplistic for plants (Grant 1975; Yamplosky and Yamplosky 1922; Fryxell 1957), it is useful for animals, which show much less diversity (Ghiselin 1969, 1974; Giese and Pearse 1974, 1975a, 1975b).

In relation to the above scheme, the problems of sex allocation may be stated as follows.

1. For a dioecious species, what is the equilibrium sex ratio (proportion of $\delta$ in a clutch) maintained by natural selection?

2. For a sequential hermaphrodite, what is the equilibrium sex order $(\hat{\sigma} \rightarrow q$, $\left.q \rightarrow \delta^{*}\right)$ and time of sex change?

3. For a simultaneous hermaphrodite, what is the equilibrium allocation of resources to male versus female function in each breeding season?

4. Under what conditions are the various states of hermaphroditism or dioecy evolutionarily stable? (e.g., when does selection favor genes for protandry over dioecy?) When is a mixture of sexual types stable?

5. Under what conditions does selection favor the ability of an individual to alter its allocation to male versus female function in response to particular environmental conditions?

These problems are very similar to one another in that each involves working out an equilibrium under natural selection where the possible genotypes have a different

* This paper is dedicated to W. D. Bunker, whose standards of excellence I can only admire.

Am. Nat. 1979. Vol. 113, pp. 465-480.

(C) 1979 by The University of Chicago. $0003-0147 / 79 / 1304-0001 \$ 01.30$ 
genetic contribution through male versus female function. Of course, answers to the questions must consider the biology of the organisms - growth, morphology, mortality, competition (interspecific and intraspecific), predation, patchiness in the environment, etc., as well as possible genetic factors (inbreeding, gene flow, etc.). The equilibria may also differ depending upon the form of inheritance (e.g., parentoffspring conflict over sex ratio--Trivers 1974; Trivers and Hare 1976; Alexander and Sherman 1977; Autosomal-sex chromosome conflict over sex ratio-Shaw 1958; Hamilton 1967).

The theoretical approach I use solves for a genetic equilibrium in a population. Maynard Smith (1976) talked of the equilibrium value of a trait of interest as an Evolutionarily Stable Strategy (or an ESS). An ESS is simply as follows. Suppose we have a population of individuals who have some attribute $X$. We introduce into this population a rare genotype with attribute $\hat{X}$ (where $\hat{X} \neq X$ ) and see whether the $\hat{X}$ individuals are selected for or against (i.e., does the rare mutant spread?). If for some character of interest (e.g., primary sex ratio), there exists an $X$ such that all deviants are selected against, $X$ is termed an ESS. The classical example is selection on the primary sex ratio (Fisher 1930) where the ESS is one-half males at conception in the simplest case. Thus a rare genotype producing an altered sex ratio in such a population is selected against. As will be shown, the ESS idea is a very useful way of asking such questions as: "At what age do we expect a cohort of protandrous shrimp to change from $\delta$ to $q$ ?" The answer is, "At an age when, under the prevailing demographic and growth conditions, genotypes that switch at some other age contribute less genetic material to future generations." Constructed this way, the models of population genetics can be used to provide answers. It is also possible that the ESS is not a single action (pure strategy) but is some array of actions, each of which takes place with some probability (mixed strategy). Finally, if the environment is nonuniform, the ESS may consist of a set of actions, with a particular one appropriate for a particular patch.

My treatment of various aspects of sex allocation theory is motivated by a rather interesting pattern which has emerged from recent modeling efforts in this area (MacArthur 1965; Leigh et al. 1976; Charnov et al. 1976). MacArthur (1965) showed in a discrete generation model that natural selection favors females who control their sex ratio, clutch size, and allocation of resources to offspring to maximize the product of the sons alive at breeding age times the daughters. His argument used an optimality model, but Spieth (1974) showed that the result follows from a simple genetic model. Charnov et al. (1976) used a discrete generation model to show that for a simultaneous hermaphrodite the equilibrium allocation of resources to male versus female function again maximizes the product of the fitness achieved through male function times that achieved through female function. These sex allocation equilibria may be redescribed as follows: Selection favors a change in allocation (e.g., sex ratio, resources, etc.) if and only if the percent gain in fitness through one sex function exceeds the percent loss through the other. Framed in this way, the equilibria correspond very nicely to the following result which Leigh et al. (1976, p. 3656) derived for a sequential hermaphrodite. They showed that, "in a nearly stable population of nearly constant age composition, selection favors a rare mutant (which alters its age of transform) if and only if it increases the prospective reproduction of 
its newborn while they are members of one sex by a percentage exceeding the percentage loss to the other sex." They also noted that this percentage result applied to the sex ratio with overlapping generations.

In this paper, I consider three problems in sex allocation; (i) natural selection when the population consists of a mixture of hermaphrodites and males or females, (ii) selection on a sex-ratio gene which affects progeny sex ratio at every age of the mother, and (iii) sex ratio in a variable environment (under parental control). Where appropriate, I assume overlapping generations (with no restrictions on population growth).

For the three models proposed here, the pattern mentioned above continues to hold. This is due to the following result (which has been known for a long time in the two-sex, single-locus, discrete-generations model, Bodmer and Parsons [1960]).

In order to find the ESS, we need a measure of fitness for a rare mutant adequate to tell us if it is being selected for or against. For the three models proposed here, this fitness measure will be shown to be of the form $W=\hat{m} / m+\hat{f} / f$, where

$\hat{m}=$ fitness of rare heterozygote through male function,

$m=$ fitness of common homozygote through male function,

$\hat{f}=$ fitness of rare heterozygote through female function,

$f=$ fitness of common homozygote through female function.

From this simple measure follows the above results for sex allocation. If the mutant is identical to the homozygote $(\hat{m}=m, \hat{f}=f), W=2$; we may assign the homozygote a fitness of 2 . The mutant is favored if $W>2$ or if $\hat{m} / m+\hat{f} / f>2$. If we write $\hat{m}=m+\Delta m, \hat{f}=f+\Delta f$; the rule becomes $\Delta m / m+\Delta f / f>0$, or (in words): Selection favors a mutant gene which alters various life history parameters if the percent gain in fitness through one sex function exceeds the percent loss through the other sex function.

Thus, the equilibrium allocation of resources to male versus female function maximizes the product of the fitness gain through male function (e.g., sons, pollen, time spent as a male) times the gain through female function (or $m \cdot f$ ). Actually, the principle proposed here is that selection will tend to maximize $m \cdot f$, both in the choice of sexual states (e.g., hermaphroditism versus dioecy) and in the allocation to male versus female function within a sexual state.

This paper is the first in a series on how natural selection operates on sex allocation. The first two models will assume overlapping generations - my purpose is simply to establish that the equilibrium conditions for a complex life history do indeed satisfy the above result. Later papers will use the formalism to propose and test some particular hypotheses. The third model reduces to a discrete generation problem. Here I discuss in more detail some of the biology behind the model, present some specific predictions, and briefly review existing data.

\section{A POPUlation AS A MIXTURE OF SEXUAL TYPES}

Charlesworth and Charlesworth (1973) developed a model for natural selection with two sexes and overlapping generations, while Leigh et al. (1976) considered selection with sequential hermaphroditism (also overlapping generations). In this 
section I will generalize these efforts (at least for the initial spread of a rare gene) by considering a population consisting of a mixture of hermaphrodites, males and females. Such populations are known to exist among plants (Lewis 1941; Lloyd 1974a, 1974b, 1975; Charnov et al. 1976; Yamplosky and Yamplosky 1922) and are variously distributed among invertebrates (Fox 1972; Ghiselin 1974; Giese and Pearse 1974, 1975a, 1975b).

For simplicity, I recognize five sexual types (,$+ \hat{\sigma}$, three hermaphrodites) but the method is not limited to them. The basic question is as follows. Consider a population homozygous at a locus of interest $(A A)$ where the genotype produces at conception a certain fraction of $\delta, q$ and various hermaphrodites. Each sexual type has a particular life table, and a specified age-fertility pattern (for hermaphrodites this is production of both sperm and eggs). Introduce into this population a rare mutant $(a)$ which alters one to many life-history parameters (e.g., fraction of each type, life table, age-fertility pattern). The question of interest is: What are the conditions under which this mutant is selected for or against? The model uses the following assumptions and definitions.

Begin with the following definitions for genotype $A A$.

$i=$ age of an individual, $i=0,1,2, \ldots, \beta_{j}$;

$j=$ sexual type of an individual,

$1=$ male,

$2=$ female,

$3=$ protogynous hermaphrodite (individual is a female until age $t_{3}$, and a male from $t_{3}+1$ until $\left.\beta_{3}\right)$,

$4=$ protandrous hermaphrodite $\left(\delta: i=0 \rightarrow t_{4}\right.$, o : $\left.i=t_{4}+1 \rightarrow \beta_{4}\right)$,

$5=$ simultaneous hermaphrodite, producing both sperm and eggs in each breeding season;

$b_{i j}=$ eggs produced by an individual of sexual type $j$ at age $i\left(b_{i j}=0\right.$ if individual is acting as a male at age $\left.i, b_{0 j}=0\right)$;

$Q_{i j}=$ quality or relative fertility of a male of sexual type $j$ at age $i$. (If the gametes are simply released into the environment $Q_{i j}$ reflects the number of sperm [or pollen grains] released by the individual, compared to the number released by some standard individual. $Q_{i j}$ can be measured on the interval $[0,1]$.$) ;$

$p_{i j}=$ survival rate from age $i$ to $i+1$ for an individual of sexual type $j$;

$q_{k}=$ proportion of the eggs (of genotype $A A$ ) which develop into sexual type $k$ $(k=1,2,3,4$, or 5$), \sum q_{k}=1$;

$n_{i j}(T)=$ number of individuals of age $i$ of sexual type $j$ at time $T$;

$l_{i j}=$ probability a newborn of sexual type $j$ is alive at age $i=\prod_{h=0}^{i-1} P_{h j}, l_{0 j}=1$.

Since all eggs produced are assumed to be fertilized, the dynamics of a population consisting only of genotype $A A$ is given by the following set of equations.

$$
\begin{gathered}
n_{0 k}(T+1)=q_{k} \sum_{j} \sum_{i}\left[n_{(i-1) j}(T) \cdot p_{(i-1) j} \cdot b_{i j}\right], \quad \text { for } \quad k=1 \rightarrow 5 ; \\
n_{i j}(T+1)=n_{(i-1) j}(T) \cdot p_{(i-1) j}, \quad \text { for } \quad i=1 \rightarrow \beta_{j}, j=1 \rightarrow 5 .
\end{gathered}
$$


This is a fairly large set of linear difference equations (there are $5+\beta_{1}+\beta_{2}+$ $\beta_{3}+\beta_{4}+\beta_{5}$ of them) but its form is rather simple, being similar to various age-parity models in human demography (Keyfitz 1968). If the set of equations is written in matrix form, the associated matrix is almost always nonnegative, irreducible and primitive (Keyfitz 1968). I restrict attention to these cases, since this insures that the population approaches a stable age-type distribution where it is changing at a constant exponential rate (i.e., $N(T+1)=\lambda_{1} \cdot N(T)$ where $N$ is the total population size). Two facts are useful, considering this population in stable age-type distribution. First, the characteristic equation (which is needed to extract the dominant eigenvalue $-\lambda_{1}$ ) has a fairly simple form. It is most easily found by the method in Wilson and Bossert (1971, p. 117) for deriving the classic Euler equation. Applying their method (see the Appendix for this derivation) to equation set (1) yields

$$
1=\sum_{j} \sum_{i}\left[l_{i j} \cdot b_{i j} \cdot q_{j} / \lambda_{1}^{i}\right], \quad i \geq 1
$$

The second useful piece of information is that in stable age-type distribution, the following ratio is a constant (independent of time, $T$ ):

$$
\frac{\sum_{j} \sum_{i}\left[n_{i j}(T) \cdot b_{i j}\right]}{\sum_{j} \sum_{i}\left[n_{i j}(T) \cdot Q_{i j}\right]}, \quad i \geq 1 .
$$

This constant (call it $R^{*}$ ) may also be developed using the simple method of Wilson and Bossert (1971). It is seen to have the form

$$
R^{*}=\frac{\sum_{j} \sum_{i}\left[l_{i j} \cdot b_{i j} \cdot q_{j} / \lambda_{1}^{i}\right]}{\sum_{j} \sum_{i}\left[l_{i j} \cdot Q_{i j} \cdot q_{j} / \lambda_{1}^{i}\right]}, \quad i \geq 1 .
$$

Consider now a rare gene $a$ introduced into this population of $A A$. When $a$ is rare, we need only consider the heterozygote $A a$ (if the heterozygote differs from $A A$ ) to know if $a$ is being selected for or against. Define for $A a$ the same parameters as for $A A$, but put a " " above them (i.e., $\hat{p}_{i j}$ ). The $\hat{n}_{0 j}$ individuals (newborn $A a$ ) come from two sources. Virtually all of the eggs produced by the $A a$ genotype are fertilized by $A A$ produced sperm (thus one-half of these zygotes are $A a$ ). Of the eggs produced by $A A$ individuals, some small fraction $(\varepsilon)$ will be fertilized by $A a$ produced sperm, and one-half of these zygotes will be $A a$. We ignore the $A A$ individuals contributed by these matings as they are negligible compared to those from $A A \times A A$ matings.

The dynamics of $a$ when rare is given by the following set of equations (to a reasonable approximation):

$$
\begin{aligned}
& \hat{n}_{0 k}(T+1) \simeq \frac{\hat{q}_{k}}{2}\left[\sum_{j} \sum_{i}\left(\hat{n}_{(i-1) j}(T) \cdot \hat{p}_{(i-1) j} \cdot \hat{b}_{i j}\right)+\varepsilon \sum_{j} \sum_{i}\left(n_{(i-1) j}(T) \cdot p_{(i-1) j} \cdot b_{i j}\right)\right], \\
& \hat{n}_{i j}(T+1)=\hat{n}_{(i-1) j}(T) \cdot \hat{p}_{(i-1) j}, \quad \text { for } \quad k=1 \rightarrow 5 ;
\end{aligned}
$$


The $\varepsilon$ may be developed as follows - it is the ratio of the total male fertility of the $A a$ individuals to the $A A$ (to a reasonable approximation), or

$$
\varepsilon \simeq \frac{\sum_{j} \sum_{i}\left[\hat{n}_{i j}(T+1) \cdot \hat{Q}_{i j}\right]}{\sum_{j} \sum_{i}\left[n_{i j}(T+1) \cdot Q_{i j}\right]}=\frac{\sum_{j} \sum_{i}\left[\hat{n}_{(i-1) j}(T) \cdot \hat{p}_{(i-1) j} \cdot \hat{Q}_{i j}\right]}{\sum_{j} \sum_{i}\left[n_{(i-1) j}(T) \cdot p_{(i-1) j} \cdot Q_{i j}\right]}, \quad i \geq 1 .
$$

If $(6)$ is substituted into (5), recalling the definition of $R^{*}$ (eq. [4]), we have

$$
\begin{aligned}
& \hat{n}_{0 k}(T+1) \simeq \frac{\hat{q}_{k}}{2} \sum_{j} \sum_{i} \hat{n}_{(i-1) j}(T)\left(\hat{b}_{i j}+R^{*} \cdot \hat{Q}_{i j}\right) \hat{p}_{(i-1) j}, \quad \text { for } \quad k=1 \rightarrow 5 ; \\
& \hat{n}_{i j}(T+1)=\hat{n}_{(i-1) j}(T) \cdot \hat{p}_{(i-1) j}, \quad \text { for } \quad i=1 \rightarrow \beta_{j}, j=1 \rightarrow 5 .
\end{aligned}
$$

Equation sets (1) and (7) have a very similar form. The dynamics of the $a$ allele (when rare) depends upon the dominant eigenvalue $(\lambda)$ associated with (7). If $\lambda>\lambda_{1}$, the $a$ gene is increasing in frequency; if $\lambda<\lambda_{1}$, it is being selected against. The $\lambda$ is given by largest positive root of the characteristic equation of (7), which is seen to have the following form (derived in the Appendix):

$$
2=\frac{\sum_{j} \sum_{i}\left[\hat{l}_{i j} \cdot \hat{b}_{i j} \cdot \hat{q}_{j} / \lambda^{i}\right]}{\sum_{j} \sum_{i}\left[l_{i j} \cdot b_{i j} \cdot q_{j} / \lambda_{1}^{i}\right]}+\frac{\sum_{j} \sum_{i}\left[\hat{l}_{i j} \cdot \hat{Q}_{i j} \cdot \hat{q}_{j} / \lambda^{i}\right]}{\sum_{j} \sum_{i}\left[l_{i j} \cdot Q_{i j} \cdot q_{j} / \lambda_{1}^{i}\right]}, \quad i \geq 1 .
$$

Equation (8) may be written as

$$
2=\frac{\hat{M}(\lambda)}{M\left(\lambda_{1}\right)}+\frac{\hat{F}(\lambda)}{F\left(\lambda_{1}\right)} .
$$

For any fixed life histories, (8) may be used to extract $\lambda$. If our interest is simply in whether or not $a$ is being selected for, the following technique is useful. Suppose (9) is rewritten as

$$
W\left(\lambda^{*}\right)=\frac{\hat{M}\left(\lambda^{*}\right)}{M\left(\lambda_{1}\right)}+\frac{\hat{F}\left(\lambda^{*}\right)}{F\left(\lambda_{1}\right)}, \quad \text { (replace } \lambda \text { with } \lambda^{*}, \text { a variable). }
$$

It is easy to show that $W\left(\lambda^{*}\right)$ is a decreasing function of $\lambda^{*}$. Of course, the $\lambda$ to be compared with $\lambda_{1}$ is that associated with $W=2$. In (10), set $\lambda^{*}=\lambda_{1}$. It follows that if $\lambda>\lambda_{1} \Leftrightarrow W>2$ (or if $\lambda<\lambda_{1} \Leftrightarrow W<2$ ). To determine whether $a$ is being selected for or against, $W\left(\lambda_{1}\right)$ is a sufficient parameter-we need only know if it is $>$ or $<2$.

Note further that these results do not require that the population be growing in size--thus they clearly apply to density dependent models.

G. C. Williams has pointed out to me that the ESS results require that mutant types be selected against with a force greater than drift or mutation pressure. Otherwise (e.g., in sex ratio) a too-many-daughters genotype may be rescued by a too-many-sons genotype that restores the sex ratio to equilibrium.

\section{SEX RATIO SELECTION WITH AGE STRUCTURE}

The model considers an autosomal gene which affects sex ratio at every age for a female and asks for a set of sex ratios such that any rare mutant with a different set is 
selected against. It is a version of a model originally proposed by Charlesworth (1978). Since we deal with only males and females, a simplification in notation is useful.

Imagine a very large population, homozygous $(A A)$ at an autosomal locus, and define, as follows, for this population $(i=0,1,2, \ldots$ where 0 is conception):

$\lambda_{1}=$ finite rate of increase for population of $A A$,

$n_{i}(t)=$ number of $q$ of age $i$ at time $t$,

$b_{i}=$ clutch size for an age $i$,

$p_{i}=$ survival rate from age $i$ to $i+1$ for a $q$,

$l_{i}=$ probability a + is alive at age $i$,

$r_{i}=$ proportion of $b_{i}$ which are ${ }^{t}$ (the sex ratio),

$Q_{i}=$ relative fertility of an age $i \hat{\delta}$, defined as before.

In an analogous way, define $n_{i}^{*}(t), p_{i}^{*}, l_{i}^{*}$ for the males in this population. Since births depend only upon the females present, $\lambda_{1}$ is given by the usual stable age equation

$$
\sum \lambda_{1}^{-i} l_{i} b_{i}\left(1-r_{i}\right)=1 .
$$

Let $\bar{r}$ be the average sex ratio. Considering the population in stable age distribution, $\bar{r}$ may be written as:

$$
\bar{r}=\left(\sum \lambda_{1}^{-i} l_{i} b_{i} r_{i}\right) /\left(\sum \lambda_{1}^{-i} l_{i} b_{i}\right) .
$$

Also useful is the fact that in stable age distribution the following ratio is a constant independent of time. (Note the close correspondence to $R^{*}$.)

$$
\begin{aligned}
R & =\frac{\sum n_{i}(t) b_{i}\left(1-r_{i}\right)}{\sum n_{i}^{*}(t) Q_{i}}=\frac{(1-\bar{r}) \sum \lambda_{1}^{-i} l_{i} b_{i}\left(1-r_{i}\right)}{\bar{r} \sum \lambda_{1}^{-i} l_{i}^{*} Q_{i}} \\
& =\frac{1-\bar{r}}{\bar{r}}\left(\frac{1}{\sum \lambda_{1}^{-i} l_{i}^{*} Q_{i}}\right) .
\end{aligned}
$$

We introduce into this population a mutant $a$ which alters one to several life history parameters. When the $a$ allele is rare, only $A a$ need be considered ( $A a$ individuals essentially mate only with $A A$ ). Denote the life history parameters of the $A a$ with a "^" The dynamics of $A a$ is given by the following set of difference equations where ? and ?' refer to yet unspecified functions and where $i$ extends over all $q$ and $\delta$ age classes.

$$
\begin{aligned}
\hat{n}_{0}(t+1) & \simeq ?, \\
\hat{n}_{0}^{*}(t+1) & \simeq ?^{\prime}, \\
\hat{n}_{i}(t+1) & =\hat{n}_{(i-1)}(t) \hat{p}_{i-1}, \\
\hat{n}_{i}^{*}(t+1) & =\hat{n}_{(i-1)}^{*}(t) \cdot \hat{p}_{i-1}^{*} .
\end{aligned}
$$

The ? may be developed as follows. Age zero $\mathrm{Aa}$ q come from two sources. $A a$ + mate essentially only with $A A \hat{\delta}$, and half of the resulting offspring are $A a$. Some small fraction $\varepsilon$ of the $A A+$ are mated to $A a \hat{\delta}$, and half of these offspring are $A a$. We 
may thus write

$$
\hat{n}_{0}(t+1) \simeq \frac{\varepsilon}{2}\left[\sum n_{(i-1)}(t) p_{(i-1)} b_{i}\left(1-r_{i}\right)\right]+\frac{1}{2}\left[\sum \hat{n}_{(i-1)}(t) \hat{p}_{(i-1)} \hat{b}_{i}\left(1-\hat{r}_{i}\right)\right] .
$$

The $\varepsilon$ is of the form

$$
\varepsilon \simeq \frac{\sum \hat{n}_{i}^{*}(t+1) \hat{Q}_{i}}{\sum n_{i}^{*}(t+1) Q_{i}} \quad \text { (to a reasonable approximation). }
$$

If (16) is substituted into (15), recalling both the stable age assumption for the $A A$ population and (13), we obtain

$$
\hat{n}_{0}(t+1) \simeq \frac{R}{2}\left(\sum \hat{n}_{(i-1)}^{*}(t) \hat{p}_{(i-1)}^{*} \hat{Q}_{i}\right)+\frac{1}{2}\left[\sum \hat{n}_{(i-1)}(t) \hat{p}_{(i-1)} \hat{b}_{i}\left(1-\hat{r}_{i}\right)\right]
$$

A similar argument for ?' (conception of sons) produces

$$
\hat{n}_{0}^{*}(t+1) \simeq\left(\frac{1}{2 \sum \lambda_{1}^{-i} l_{i}^{*} Q_{i}}\right)\left(\sum \hat{n}_{(i-1)}^{*}(t) \hat{p}_{(i-1)}^{*} \hat{Q}_{i}\right)+\frac{1}{2}\left(\sum \hat{n}_{(i-1)}(t) \hat{p}_{(i-1)} \hat{b}_{i} \hat{r}_{i}\right) .
$$

If (17) and (18) are substituted into (14), the result is a set of linear difference equations which describes the dynamics of $A a$ when rare. If we represent the set as matrix multiplication, the projection matrix can be shown to be primitive, given certain regularity conditions which will normally be satisfied in real situations. We can therefore apply the Perron-Frobenius theorem which implies that $\lambda$ (the dominant eigenvalue of the projection matrix) is unique, real and positive. If $\lambda_{>} \lambda_{1}$, the $A a$ are increasing relative to the $A A$; if $\lambda \leq \lambda_{1}$, the $A a$ are being selected against. To get this characteristic equation, note that if the $A a$ are in stable age distribution, we may write:

$$
\begin{gathered}
\hat{n}_{i}(t+1)=\hat{n}_{0}(t+1) \lambda^{-i} \hat{l}_{i}, \\
\hat{n}_{i}^{*}(t+1)=\hat{n}_{0}^{*}(t+1) \lambda^{-i} \hat{l}_{i}^{*} .
\end{gathered}
$$

If these are substituted into (17) and (18), the following result (dropping the time index):

$$
\begin{aligned}
& \hat{n}_{0}=\hat{n}_{0} c_{11}+\hat{n}_{0}^{*} c_{12}, \\
& \hat{n}_{0}^{*}=\hat{n}_{0} c_{21}+\hat{n}_{0}^{*} c_{22},
\end{aligned}
$$

where

$$
\begin{array}{llrl}
c_{11} & =\frac{1}{2}\left[\sum \lambda^{-i} \hat{l}_{i} \hat{b}_{i}\left(1-\hat{r}_{i}\right)\right], & c_{12} & =\frac{R}{2}\left(\sum \lambda^{-i} \hat{l}_{i}^{*} \hat{Q}_{i}\right), \\
c_{21} & =\frac{1}{2}\left(\sum \lambda^{-i} \hat{l_{i}} \hat{b}_{i} \hat{r}_{i}\right), & c_{22} & =\left(\sum \lambda^{-i} \hat{l}_{i}^{*} \hat{Q}_{i}\right) /\left(2 \sum \lambda_{1}^{-i} l_{i}^{*} Q_{i}\right) .
\end{array}
$$

The characteristic equation may be written in terms of the $c_{j k}$ as follows (eliminating $n_{0}$ and $n_{0}^{*}$ from [21])

$$
1=c_{11}+c_{22}+c_{21} \cdot c_{12}-c_{11} \cdot c_{22}
$$


Equation (22) is necessary for the consistency of the equations (21). It is identical to the characteristic equation derived by Charlesworth (1978). Equation (22) may be solved for $\lambda$, which can then be compared to $\lambda_{1}$. Again, there is a much easier way to make this comparison. Define $w\left(\lambda^{*}\right)$ to be a function of $\lambda^{*}$ where $\lambda^{*}$ replaces $\lambda$. It is easy to show that (22) is a decreasing function of $\lambda^{*}$. It follows that $w(\lambda)=1$; $w\left(\lambda^{*}>\lambda\right)<1 ; w\left(\lambda^{*}<\lambda\right)>1$. In particular, $\lambda>\lambda_{1} \Leftrightarrow w\left(\lambda_{1}\right)>1$ and $\lambda<\lambda_{1} \Leftrightarrow$ $w\left(\lambda_{1}\right)<1$. Thus $w\left(\lambda_{1}\right)$ is a fitness measure, adequate to tell us if $a$ is being selected for or against. One assumption will make the substitution of $c_{j k}$ in (22) easier; the interest is in a gene which affects sex ratio and the various life history parameters for the mother. If the gene does not affect the parameters for the father or son (i.e., all males follow the same life history) $\hat{l}_{i}^{*}=l_{i}^{*}$ and $\hat{Q}_{i}=Q_{i}$. With this in mind, recalling (11), (12), and (13), we have after a bit of algebra:

$$
w^{*}\left(\lambda_{1}\right)=\left[4 w\left(\lambda_{1}\right)-2\right]=\frac{\sum \hat{l}_{i} \lambda_{1}^{-i} \hat{b}_{i} \hat{r}_{i}}{\sum l_{i} \lambda_{1}^{-i} b_{i} r_{i}}+\frac{\sum \lambda_{1}^{-i} \hat{l}_{i} \hat{b}_{i}\left(1-\hat{r}_{i}\right)}{\sum \lambda_{1}^{-i} l_{i} b_{i}\left(1-r_{i}\right)} .
$$

Equation (23) has a very intuitive interpretation-it is the sons born to an $A a$ female over her lifetime (discounted for population growth) divided by the sons of an $a a$ female plus the same for the daughters produced by each. Thus the form of the equation is, as before, $\hat{m} / m+\hat{f} / f$, with appropriate definition of fitness through male and female function.

\section{PARENTAL CONTROL OF SEX RATIO IN A PATCHY ENVIRONMENT:} PARASITIC HYMENOPTERA

Most hymenoptera have haplodiploid sex determination-males develop from unfertilized eggs, females from fertilized. The uniqueness of haplodiploidy is that the mother can easily control the sex of her offspring. Here I develop a model for how a female wasp should alter her sex ratio in response to change in a major environmental variable. The resulting theory is again in the general form $\hat{m} / m+\hat{f} / f=W$. The model makes a set of simple and testable predictions, some of which are supported by existing literature. Since the patterns have a long history in the literature, a brief review of the biology will serve as an introduction.

One of the most striking patterns of sex ratio alteration is found with solitary parasites attacking a host population in which the hosts are of various sizes. It is typical for the parasite to produce sons in small hosts and daughters in large hosts--at least the sex ratio tends to be biased in this direction (Chewyreuv 1913; Brunson 1934, 1937; Roberts 1933; Holdaway and Smith 1933; Clausen 1939; Assem 1971). The same pattern is present in trap-nesting bees and wasps, with more males produced in cells of small diameter (Krombein 1967). Along with this shift in sex ratio, two other patterns are important. (i) It is usual in Hymenoptera for females to be larger than males. (ii) Within a species, smaller than average females typically suffer reduced fertility (although this has not actually been demonstrated for most of the above species [Assem 1971]). Interestingly, small males may not suffer a great disadvantage in mating (at least for one species, small males had no difficulty in getting females to accept them [Assem 1971]). 


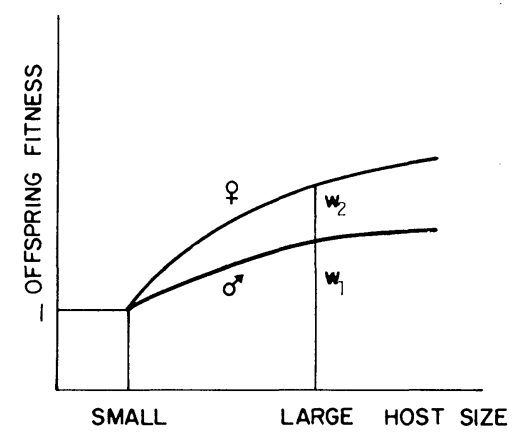

FIG. 1.-Fitness as a function of host size: The fitness of a son or daughter is a function of the host size in which they are placed. Fitness is relative within each sex. In this figure, offspring in "small hosts" have a relative fitness of one. In "large hosts," sons have a fitness $w_{1}$; daughters $w_{2}$. Since $w_{2}>w_{1}$, a daughter gains more by being raised in a large host than does a son.

Chewyreuv (1913) was perhaps the first to clearly recognize these patterns (except that relating to males). He suggested that (1) females practiced selective oviposition, laying more unfertilized eggs in small hosts, and that (2) the action represented an adaptation since daughters typically require more food than sons. Since the size of the resulting wasp is highly related to the size of the brood host, a small host is thus not as good as a large host for producing a daughter. Chewyreuv's theory has been discussed by several of the authors already mentioned, and it seems clearly established that the sex ratio alteration comes about through selective fertilization by the mother. The second part of the theory has received much less attention. An almost identical theory has been proposed by Trivers and Willard (1973) for adaptive change in progeny sex ratio on the part of a female mammal.

Chewyreuv (1913) has proposed a very interesting theory. I intend to develop it further, using the ESS concept. In order to make quantitative predictions, it is necessary to consider both the distribution of host sizes and the way in which male and female fitness (survival $\times$ fertility) are related to host size. Other factors, such as host age, etc., may also prove important, but it seems necessary to first look at the behavior of a fairly simple model. This model will now be discussed.

Suppose a parasite population is presented with hosts of two sizes $(L=$ large, $S=$ small) in specified proportions. An ovipositing female is assumed to control her sex ratio in both $L$ and $S$ hosts. If $r_{1}=$ proportion of eggs laid in $S$ which are male $\left(r_{2}\right.$ for $L$ ); the ESS is $r_{1}$ and $r_{2}$ such that any female deviating from these sex ratios is selected against.

Referring to figure 1, the model makes the following assumptions.

1. All mothers are inseminated (results only slightly altered if this condition is violated).

2. Small hosts are present with frequency $P$.

3. Both sons and daughters produced in small hosts are assigned a fitness of 1 . Sons produced in large hosts have a fitness $W_{1}$, daughters $W_{2}$.

These measures are relative fitness within each sex. Thus an $L$ male inseminates $W_{1}$ females for every one female inseminated by an $S$ male. Likewise, an $L$ female lays $W_{2}$ as many eggs (on the average) as an $S$ female. These assumptions are shown in figure 
1, where the fitness functions for sons and daughters are drawn for a range of host sizes. Two of these are designated $S$ and $L$. Note that for these $W_{2}>W_{1}$.

Consider a mutant $A$ introduced into a population of mostly $a a, a$ where its female bearers alter their sex ratios to $\hat{r}_{1}$ and $\hat{r}_{2}$. The dynamics of this gene (when rare) may be developed as follows. Let the frequency of $A$ (among the males) be $\varepsilon_{1}$ (very small), the frequency of $A a_{S}$ ( $S$ refers to those derived from small hosts) and $A a_{L}$ (among the females) are $\varepsilon_{2}$ and $\varepsilon_{3}$ respectively. In order to keep the mathematics reasonably simple, I will also assume $W_{1}=1$. (The extension to $W_{1} \neq 1$ proves to be trivial and will be provided at the end of the argument.) Finally, let $q=$ frequency of $a a_{S}$ (among the females).

There are six mating combinations which occur with frequency $>\varepsilon^{2}$. Their frequency and progeny production are shown in table 1 . I have dropped all terms of order $\varepsilon^{2}$ and have ignored all $a a$ or $a$ contributed via matings involving either $A$ or $A a$. If we define the new genotype frequencies as $\varepsilon_{1}^{\prime}, \varepsilon_{2}^{\prime}$ and $\varepsilon_{3}^{\prime}$, we have (to a reasonable approximation)

$$
\varepsilon_{1}^{\prime} \simeq \# A / \# a ; \quad \varepsilon_{2}^{\prime} \simeq \frac{\# A a_{S}}{\# a a} ; \quad \varepsilon_{3}^{\prime} \simeq \frac{\# A a_{L}}{\# a a} .
$$

One fact will make the derivation of these ratios easier. The average fitness of an $a a$ is $q+(1-q) W_{2}$. In terms of sex ratio this may be written as $q+(1-q) W_{2}=$ $\left[P\left(1-r_{1}\right)+(1-P)\left(1-r_{2}\right) W_{2}\right] /\left[P\left(1-r_{1}\right)+(1-P)\left(1-r_{2}\right)\right]$. With this in mind, forming the ratios given in (24), we arrive at the following, written as a matrix multiplication.

$$
\left(\begin{array}{ccc}
0 & \frac{h}{B} & \frac{W_{2} h}{B} \\
\frac{\left(1-r_{1}\right) P\left(q+W_{2}(1-q)\right)}{D} & \frac{P F_{1}}{D} & \frac{P W_{2} F_{1}}{D} \\
\frac{(1-P)\left(1-r_{2}\right)\left(q+W_{2}(1-q)\right)}{D} & \frac{F_{2}(1-P)}{D} & \frac{F_{2}(1-P) W_{2}}{D}
\end{array}\right)\left(\begin{array}{c}
\varepsilon_{1} \\
\varepsilon_{2} \\
\varepsilon_{3}
\end{array}\right)=\left(\begin{array}{c}
\varepsilon_{1}^{\prime} \\
\varepsilon_{2}^{\prime} \\
\varepsilon_{3}^{\prime}
\end{array}\right)
$$

where

$$
\begin{aligned}
h & =\left[P \hat{r}_{1}+(1-P) \hat{r}_{2}\right] / 2, \\
B & =\left[a+W_{2}(1-q)\right]\left[r_{1} P+r_{2}(1-P)\right], \\
D & =P\left(1-r_{1}\right)+(1-P)\left(1-r_{2}\right) W_{2}, \\
F_{1} & =\left(1-\hat{r}_{1}\right) / 2, \\
F_{2} & =\left(1-\hat{r}_{2}\right) / 2 .
\end{aligned}
$$

Equation (25) is singular since the third column is a constant multiple of the second.

This makes the characteristic equation a quadratic equation of rather simple form. In terms of $a_{i j}(i=1,2,3 ; j=1,2,3)$ it is as follows.

$$
\lambda^{2}-\lambda\left(a_{22}+a_{33}\right)-a_{31} a_{13}-a_{21} a_{12}=0 \text {. }
$$

If the dominant root of 3.3 is $>1$, the mutant is being favored. (If $\lambda<1$, it is being selected against.) It is useful to examine the set of parameters which make $\lambda=1$. 
TABLE 1

Offrpring Produced by the Six Matings in the Parasite System

\begin{tabular}{|c|c|c|c|c|}
\hline \multirow[b]{2}{*}{ Females } & \multicolumn{4}{|c|}{ Males } \\
\hline & & $A \sim \varepsilon_{1}$ (frequency) & & $a \sim 1$ \\
\hline \multicolumn{5}{|l|}{ (frequency) } \\
\hline$A a_{S} \sim \varepsilon_{2} \ldots \ldots$ & & $\cdots$ & $\begin{array}{l}A a_{S} \\
A a_{L} \\
A\end{array}$ & $\begin{array}{l}\varepsilon_{2} P\left(1-\hat{r}_{1}\right) / 2 \\
\varepsilon_{2}(1-P)\left(1-\hat{r}_{2}\right) / 2 \\
\left\{\varepsilon_{2}\left[P \hat{r}_{1}+(1-P) \hat{r}_{2}\right]\right\} / 2\end{array}$ \\
\hline$A a_{L} \sim \varepsilon_{3} \ldots \ldots$ & & $\cdots$ & $\begin{array}{l}A a_{S} \\
A a_{L} \\
A\end{array}$ & $\begin{array}{l}W_{2} \varepsilon_{3} P\left(1-\hat{r}_{1}\right) / 2 \\
W_{2} \varepsilon_{3}(1-P)\left(1-\hat{r}_{2}\right) / 2 \\
\left\{\varepsilon_{3} W_{2}\left[P \hat{r}_{1}+(1-P) \hat{r}_{2}\right]\right\} / 2\end{array}$ \\
\hline$a a_{S} \sim q \ldots \ldots$ & $\begin{array}{l}A a_{S} \\
A a_{L}\end{array}$ & $\begin{array}{l}\varepsilon_{1} q P\left(1-r_{1}\right) \\
\varepsilon_{1} q(1-P)\left(1-r_{2}\right)\end{array}$ & $\begin{array}{l}a a \\
a\end{array}$ & $\begin{array}{l}q\left[P\left(1-r_{1}\right)+(1-P)\left(1-r_{2}\right)\right] \\
q\left[P r_{1}+(1-P) r_{2}\right]\end{array}$ \\
\hline$a a_{L} \sim(1-q) \ldots$ & $\begin{array}{l}A a_{S} \\
A a_{L}\end{array}$ & $\begin{array}{l}W_{2} \varepsilon_{1}(1-q) P\left(1-r_{1}\right) \\
W_{2} \varepsilon_{1}(1-q)(1-P)\left(1-r_{2}\right)\end{array}$ & $\begin{array}{l}a a \\
a\end{array}$ & $\begin{array}{l}W_{2}(1-q)\left[P\left(1-r_{1}\right)+(1-P)\left(1-r_{2}\right)\right] \\
W_{2}(1-q)\left[r_{1} P+(1-P) r_{2}\right]\end{array}$ \\
\hline
\end{tabular}

Setting $\lambda=1$ in (26), we arrive at the following after a bit of algebra.

$$
2=\frac{P\left(1-\hat{r}_{1}\right)+W_{2}(1-P)\left(1-\hat{r}_{2}\right)}{P\left(1-r_{1}\right)+W_{2}(1-P)\left(1-r_{2}\right)}+\frac{P \hat{r}_{1}+(1-P) \hat{r}_{2}}{P r_{1}+(1-P) r_{2}} .
$$

This is, of course, of the form $\hat{m} / m+\hat{f} / f$. As before, we may not consider (27) equal to 2 , but a variable measure of fitness $(W=\hat{m} / m+\hat{f} / f)$. If $W>2$, the mutant is being selected for; if $W<2$ it is being selected against.

To extend the analysis to cases where the male fitness is also a function of host size $\left(1<W_{1}<W_{2}\right)$, I argue as follows. Since $A$ are produced only by $A a$, the average fraction of matings which involve $A$ is $\sim$ (fertility of all $A$ ) $/($ fertility of all $a$ ). We must thus multiply $\varepsilon_{1}$ (fraction of males that are $A$ ) by the average fertility of an $A$ versus $a$. This ratio (call it $l$ ) is:

$$
l=\frac{\left[P \hat{r}_{1}+(1-P) \hat{r}_{2} W_{1}\right] /\left[P \hat{r}_{1}+(1-P) \hat{r}_{2} W_{1}\right]}{\left[P r_{1}+(1-P) r_{2} W_{1}\right] /\left[P r_{1}+(1-P) r_{2}\right]} .
$$

The end result is simply that instead of $a a \times A$ matings being of frequency $\varepsilon_{1}$ they happen with frequency $l \varepsilon_{1}$ - this simply multiplies $a_{21}$ and $a_{31}$ in (25) and (26) by $l$. Setting $\lambda=1$ as before shows that, for this case, the analogue to (27) is

$$
2=\frac{P\left(1-\hat{r}_{1}\right)+W_{2}(1-P)\left(1-\hat{r}_{2}\right)}{P\left(1-r_{1}\right)+W_{2}(1-P)\left(1-\hat{r}_{2}\right)}+\frac{P \hat{r}_{1}+(1-P) \hat{r}_{2} W_{1}}{P r_{1}+(1-P) r_{2} W_{1}} .
$$

Again the form is $\hat{m} / m+\hat{f} / f$-all of the former arguments still hold (considering [28] to be a fitness measure, instead of equal to 2).

It should be noted that extension of the above results to more than two host sizes is straightforward - my restriction to two sizes is based on the desire to produce an 


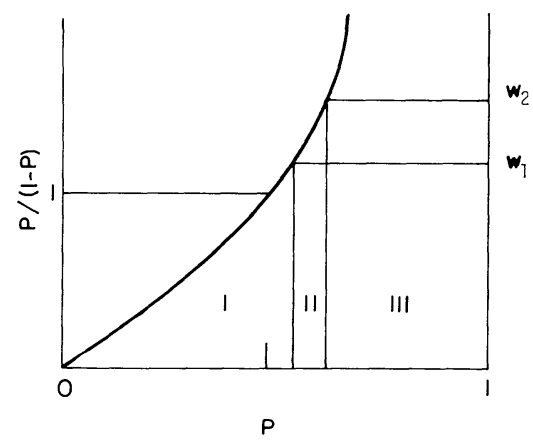

FIG. 2.-The ESS sex ratios under the host size model: Analysis of the model shows that $P$ is divided into regions I, II, III given by $w_{1}$ and $w_{2}$. In each region a different sex ratio rule applies.

easily tested model. In terms of an ESS analysis, the two-size model contains only three parameters $\left(W_{1}, W_{2}, P\right)$, all of which are easily varied in the lab.

An ESS analysis for (28) looks for $r_{1}$ and $r_{2}$ such that $(28) \leq 2$ for all possible $\hat{r}_{1}$ and $\hat{r}_{2}$. If we examine how fitness changes with $\hat{r}_{1}$ and $\hat{r}_{2}\left(\right.$ i.e., $\partial W / \partial \hat{r}_{1}, \partial W / \partial \hat{r}_{2}$ ), the results assume a fairly simple form. The ESS sex ratios depend on $W_{1}, W_{2}$, and $P$ in a fairly simple way. There are three regions of $P$, and a different sex ratio rule applies in each one. (Recall that $W_{2}>W_{1}$.) These regions are shown in figure 2.

Region I: $P /(1-P)<W_{1} ;$ sex ratio results, $r_{1}=1, r_{2}=\frac{W_{1}(1-P)-P}{2 W_{1}(1-P)}$.

Region II: $W_{1}<P /(1-P)<W_{2}$; sex ratio results, $r_{1}=1, r_{2}=0$.

Region III: $P /(1-P)>W_{2}$; sex ratio results, $r_{1}=\frac{P+(1-P) W_{2}}{2 P}, r_{2}=0$.

In region I (which always includes $P \leq \frac{1}{2}$ ), the female fitness $\left(W_{2}\right)$ plays no role; in region III, the male fitness $\left(W_{1}\right)$ plays no role. In general, the results are as suggested by Chewyreuv's theory, but it is important to note that they rest on $W_{2}>W_{1}$. It is not that small hosts are better for producing sons than daughters (a between sex comparison) but that sons gain less in fitness by being raised in a large host, relative to the gain of a daughter (a within sex comparison). At present I am testing this model (with a host-parasite system) in a semiquantitative way. It is easy to vary $P$; and $W_{1}$ and $W_{2}$ may be qualitatively varied by varying host size.

The model provides an answer to one somewhat puzzling aspect of Chewyreuv's (1913) data. As discussed by Assem (1971): "A most interesting result was that it does not seem to be the absolute size of the host alone, but some relative measurements as well that play a role, though Chewyreuv does not conclude so explicitly. When he offered, again in an irregular order, (host) pupae of Sphinx ligustri (large) and those of Pieris brassicae (small), the former produced females and the latter males. If, however, the Pieris pupae were alternated with those of Vanessa levana (still smaller) the same wasps produced an offspring of females in the former and males in the latter." This is exactly the outcome predicted under the present model. 
SUMMARY

R. A. Fisher (1930) was perhaps the first to realize that the key to sex ratio evolution lay in the almost trivial fact that (under diploidy) everyone has one mother and one father; that in terms of autosomal genes males and females contribute equally to any zygote formed. This paper shows that his observation proves a useful key to a host of other sex related problems. It is for this intuitive reason that fitness measures for the alteration of sex function are often of the general form $W=\hat{m} / m+$ $\hat{f} / f$. In such a measure male and female function are assigned equal weight. It is somewhat surprising that this notion continues to hold under haplodiploidy (at least from the mother's viewpoint).

There is much that this paper has ignored-inbreeding, fluctuating or stochastic environments, etc. A treatment of many of these is much beyond me. It will be quite interesting to know how well the $\hat{m} / m+\hat{f} / f$ notion holds up to alterations in the basic models proposed here.

\section{ACKNOWLEDGMENTS}

Earlier drafts of these ideas were critically reviewed by Brian Charlesworth, James Crow, and E. G. Leigh, Jr. They are not responsible for the presentation, but it is much clarified due to their efforts, which I gratefully acknowledge. I thank J. Werren and J. Van Den Assem for much discussion on wasp sex ratios. I especially wish to thank James Bull for several years of free give and take in the area of sex allocation theory. Finally, Richard Shaw and J. D. Mohler (1953) first taught me to think about sex ratio problems - it is an intellectual debt I am happy to acknowledge. This work was supported in part by National Science Foundation grant DEC-76-83011.

\section{APPENDIX}

\section{The Characteristic Equations}

This appendix will derive equations (2) and (8). If the population of $A A$ is in stable age-type distribution, we may write $n_{0 j}(\tau-x)=n_{0 j}(\tau) / \lambda_{1}^{x}$. Thus we may also write $n_{i j}(\tau)=$ $n_{0 j}(\tau-i) \cdot l_{i j}=n_{0 j}(\tau) \cdot l_{i j} / \lambda_{1}^{i}$, but from equations $(1)$

Thus

$$
n_{0 k}(T+1)=q_{k} \cdot \sum_{j} \sum_{i} n_{i j}(T+1) \cdot b_{i j}
$$

$$
n_{0 k}(T+1)=q_{k} \cdot \sum_{j} \sum_{i}\left[n_{0 j}(T+1) \cdot l_{i j} \cdot b_{i j} / \lambda_{1}^{i}\right] .
$$

Let $N_{0}(T+1)$ be the total number of newborns at $T+1$, then for $j=1,2, \ldots, 5$, $n_{0 j}(T+1)=q_{j} \cdot N_{0}(T+1)$ and (A2) may be written as

or

$$
N_{0}(T+1) \cdot q_{k}=q_{k} \sum_{j} \sum_{l}\left[N_{0}(T+1) \cdot q_{j} \cdot l_{i j} \cdot b_{i j} / \lambda_{1}^{i}\right]
$$

$$
1=\sum_{j} \sum_{i}\left[l_{i j} \cdot b_{i j} \cdot q_{j} / \lambda_{1}^{i}\right]
$$

which is of course (2).

To get equation (8), consider the $A a$ population in stable age-type distribution. We may write

$$
\hat{n}_{0 j}(\tau-x)=\hat{n}_{0 j}(\tau) / \lambda^{x}
$$


We may also write $\hat{n}_{i j}(\tau)=\hat{n}_{0 j}(\tau-i) \cdot \hat{l}_{i j}=\hat{n}_{0 j}(\tau) \cdot \hat{l}_{i j} / \lambda^{i}$ but $\hat{n}_{0 j}(\tau)=\hat{q}_{j} \cdot N_{0}(\tau)$ where $N_{0}(\tau)=$ total newborn $A a$ at $\tau$. The first equation in set (7) may be written as

$$
\hat{q}_{k} \cdot N_{0}(T+1)=\left(\hat{q}_{k} / 2\right) \sum_{j} \sum_{i}\left[\hat{q}_{j} \cdot N_{0}(T+1)\left(\hat{b}_{i j}+R^{*} \cdot \hat{Q}_{i j}\right)\right] \hat{l}_{i j} \cdot \lambda^{-i}
$$

or

$$
2=\sum_{j} \sum_{i}\left[\hat{q}_{j} \lambda^{-i} \hat{i}_{i j}\left[\hat{b}_{i j}+R^{*} \cdot \hat{Q}_{i j}\right]\right.
$$

or, using (2) and (4),

$$
2=\frac{\sum_{j} \sum_{i}\left[\hat{q}_{j} \cdot \hat{l}_{i j} \cdot \hat{b}_{i j} / \lambda^{i}\right]}{\sum_{j} \sum_{i}\left[q_{j} \cdot l_{i j} \cdot b_{i j} / \lambda_{1}^{i}\right]}+\frac{\sum_{j} \sum_{i}\left[\hat{q}_{j} \cdot \hat{l}_{i j} \cdot \hat{Q}_{i j} / \lambda^{i}\right]}{\sum_{j} \sum_{i}\left[q_{j} \cdot l_{i j} \cdot Q_{i j} / \lambda_{1}^{i}\right]} .
$$

which is (8) (since the term $\sum_{j} \sum_{i}\left[q_{j} \cdot l_{i j} \cdot b_{i j} / \lambda_{1}^{i}\right]=1$ ).

\section{LITERATURE CITED}

Alexander, R. D., and P. W. Sherman. 1977. Local mate competition and parental investment in social insects. Science 196:494-500.

Assem, J. Van Den. 1971. Some experiments on sex ratio and sex regulation in the Pteromalid Lariophagus distinguendus. Neth. J. Zool. 21:373-402.

Bodmer, W. F., and P. A. Parsons. 1960. The initial progress of new genes with various genetic systems. Heredity $15: 283-299$.

Brunson, M. H. 1934. The fluctuation of the population of Tiphia popilliavora (Rohwer) in the field and its possible causes. J. Econ. Entomol. 27:514-518.

- 1937. The influence of the instars of host larvae on the sex of progeny of Tiphia popilliavora. Science 86:197.

Charlesworth, B. 1971. Population genetics, demography and the sex ratio. Pages 345-364 in F. B. Christiansen and T. M. Fenchel, eds. Measuring selection in natural populations. SpringerVerlag, Berlin.

Charlesworth, B., and D. Charlesworth. 1973. The measurement of fitness and mutation rate in human populations. Ann. Hum. Genet. 37:175-187.

Charnov, E. L., J. Maynard Smith, and J. J. Bull. 1976. Why be an hermaphrodite? Nature 263:125-126.

Chewyreuv, I. 1913. Le rôle des femelles dans la détermination du sexe de leur descendance dans le groupe des Ichneumonides. C. R. Soc. Biol. Paris 74:695-699.

Clausen, C. D. 1939. The effect of host size upon the sex ratio of Hymenopterous parasites and its relation to methods of rearing and colonization. J. N.Y. Entomol. Soc. 47:1-9.

Fisher, R. A. 1930. The genetical theory of natural selection. Oxford University Press, Oxford.

Fox, W. W. 1972. Dynamics of exploited Pandalid shrimps and an evaluation of management models. Ph.D. thesis, University of Washington.

Fryxell, P. 1957. Mode of reproduction in higher plants. Bot. Rev. 23:135-233.

Ghiselin, M. T. 1969. The evolution of hermaphroditism among animals. Q. Rev. Biol. 44:189-208. 1974. The economy of nature and the evolution of sex. University of California Press, Berkeley.

Giese, A. C., and J. S. Pearse, eds. 1974. Reproduction of marine invertebrates. Vol. I. Academic Press, New York.

1975a. Reproduction of marine invertebrates. Vol. II. Academic Press, New York.

1975b. Reproduction of marine invertebrates. Vol. III. Academic Press, New York.

Grant, V. 1975. Genetics of flowering plants. Columbia University Press, New York.

Hamilton, W. D. 1967. Extraordinary sex ratios. Science 156:477-488.

Holdaway, F. T., and H. F. Smith. 1933. A relation between size of host puparia and sex ratio of Alysia manducator. Aust. J. Exp. Biol. Med. Sci. 10:247-259.

Keyfitz, N. 1968. Introduction to the mathematics of population. Addison-Wesley, New York.

Krombein, K. V. 1967. Trap-nesting wasps and bees. Smithsonian, Washington D.C. 
Leigh, E. G., E. L. Charnov, and R. R. Warner. 1976. Sex ratio, sex change and natural selection. Proc. Natl. Acad. Sci. USA. 73: 3656-3660.

Lewis, D. 1941. Male sterility in natural populations of hermaphrodite plants. New Phytol. 40:56-63.

Lloyd, D. G. 1974a. Female-predominant sex ratios in angiosperms. Heredity 31:35-44.

1974b. Theoretical sex ratios of dioecious and gynodioecious angiosperms. Heredity 31:11-34.

. 1975. The maintenance of gynodioecy and androdioecy in angiosperms. Genetics $45: 325-339$.

MacArthur, R. H. 1965. Ecological consequences of natural selection. Pages 388-397 in T. Waterman and H. Morowitz, eds. Theoretical and mathematical biology. Blaisdell, Lexington, Mass.

Maynard Smith, J. 1976. Evolution and the theory of games. Am. Sci. 64:41-45.

Roberts, R. A. 1933. Biology of Brachymeria fonscolombie, a hymenopterous parasite of blowfly larvae. U.S. Dept. Agr. Tech. Bull. 365.

Shaw, R. F. 1958. The theoretical genetics of the sex ratio. Genetics $93: 149-163$.

Shaw, R. F., and J. D. Mohler. 1953. The selective advantage of the sex ratio Am. Nat. 87:337-342.

Spieth, P. T. 1974. Theoretical considerations of unequal sex ratios. Am. Nat. 87:837-849.

Trivers, R. L. 1972. Parental investment and sexual selection. Pages 136-179 in B. G. Campbell, ed. Sexual selection and the descent of man. Aldine, Chicago. 1974. Parent-offspring conflict. Am. Zool. 14:249-265.

Trivers, R. L., and H. Hare. 1976. Haplodiploidy and the evolution of the social insects. Science 191:249-263.

Trivers, R. L., and D. E. Willard. 1973. Natural selection of parental ability to vary the sex ratio of offspring. Science 179:90-92.

Williams, G. C. 1975. Sex and evolution. Princeton University Press, Princeton, N.J.

Wilson, E. O., and W. H. Bossert. 1971. A primer of population biology. Sinauer, Sunderland, Mass.

Yampolsky, C., and H. Yampolsky. 1922. Distribution of sex forms in the phanerogamic flora. Biblogr. Genet. 3:1-62. 